\title{
MicroscopyPioneers \\ Pioneers in Optics: Armand Fizeau
}

From the website Molecular Expressions created by the late Michael Davidson and now maintained by Eric Clark, National Magnetic Field Laboratory, Florida State University, Tallahassee, FL 32306

eclark@magnet.fsu.edu

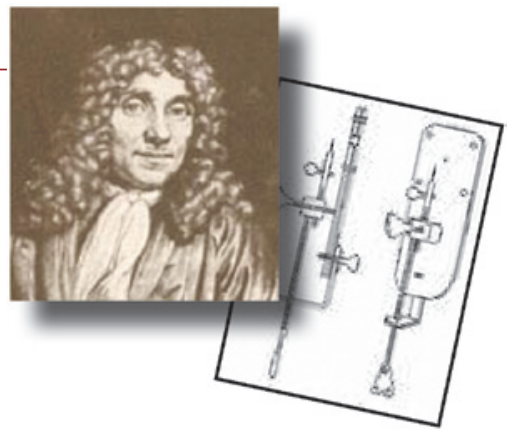

\section{Armand Fizeau}

(1819-1896)

Armand-Hippolyte-Louis Fizeau was a French physicist best known for being the first to develop a reliable experimental method of determining the speed of light on Earth. Previously, the speed of light was measured based on astronomical phenomena.

Fizeau was born in Paris on September 23, 1819. His father was a distinguished physician and professor of medicine during the Restoration period and, when he died, he left his son independently wealthy. The freedom from pecuniary concerns enabled Fizeau to pursue whatever interested him, which was primarily scientific research. He attended Stanislas College in Paris and initially intended to earn a medical degree, but he changed his mind because of ill health. Instead, Fizeau spent time traveling before returning to study with Francois Arago at the Paris Observatory.

In 1839, Fizeau became engrossed with the new daguerreotype photography and was approached by JeanBernard-Leon Foucault about his efforts to increase its

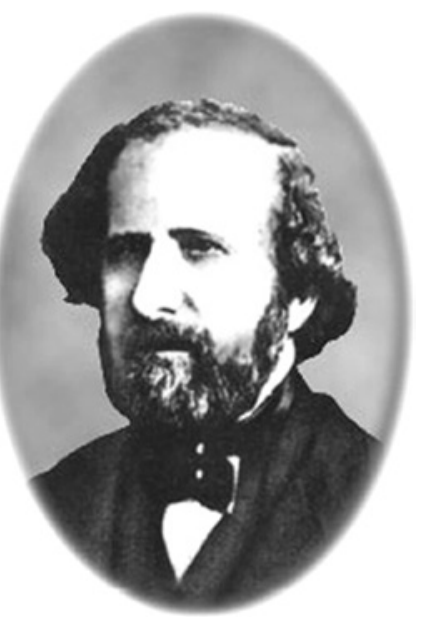
permanency. Each being intrigued by the other's work, the two decided to combine their efforts and spent much of the next ten years in scientific collaboration. Together, the French scientists developed daguerreotype photography for astronomical observations by taking the first detailed photographs of the sun's surface in 1845. However, their most important work was yet to come.

The Danish astronomer, Ole Roemer, made the first serious calculation of the speed of light in 1676. Roemer's deduction was based on his observations of the eclipses of Io, Jupiter's first moon. However, it was not until 1849 that Fizeau, working closely with Foucault, directly measured the speed of light on Earth. Fizeau used a cogwheel and a mirror located several miles apart, which were set up to allow a pulsing light beam to pass between them. By rotating the cogwheel, Fizeau was able to observe the light beam passing between the cogs of the wheel to the distant mirror and then reflected back. If he spun the wheel fast enough, he was able to obscure the reflection. This meant that the reflected light beam struck a cog. Fizeau proposed that the time it took for the wheel to move the width of one cog must be equal to the time it takes for the light beam to travel to the mirror and back to strike the cog. Knowing the rotational speed of the cogwheel, the width of one cog, and the distance to the mirror, were all that was required for Fizeau to make the simple calculation of the speed of light. His value was 313,000 kilometers per second, within 5\% of today's value.

Fizeau also studied another important phenomenon of light. He conducted experiments that demonstrated that the velocity of light is a constant, regardless of the motion of the medium it is passing through. It was previously established that light traveled at different rates through different mediums. However, prior to Fizeau's discovery, it was believed that if the medium was in motion, the velocity of the speed of light would be increased. Fizeau conducted studies in which he measured the speed of light through flowing liquids. He discovered, to his surprise, that the velocity of light did not increase by the movement of the liquids. His observations were contradictory to Newton's laws of classical mechanics and contributed to discoveries made concerning the properties of light during the twentieth century.

Other scientific contributions made by Fizeau include measuring the velocity of electricity in 1850 , describing the utilization of the condenser as a means for enhancing the efficiency of the induction coil in 1853, and conducting studies regarding the expansion of solids by heat. Due to his extensive body of research, Fizeau was elected as a member of the French Academy in 1860 and, three years later, was appointed superintendent of physics at the Polytechnic School in Paris. In 1866 the Royal Society of London awarded him the Rumford Medal. Fizeau passed away in Venteuil, France, on September 18, 1896.

Measuring the Speed of Light - Starting with Ole Roemer's 1676 breakthrough endeavors, the speed of light has been measured at least 163 times by more than 100 investigators using a wide variety of techniques. Finally in 1983, more than 300 years after the first serious measurement attempt, the speed of light was defined as being 299,792.458 kilometers per second by the Seventeenth General Congress on Weights and Measures. In general, however, (even in many scientific calculations) the speed of light is rounded to 300,000 kilometers (or 186,000 miles) per second. 


\section{Incomparable...}

\section{...and still innovating}

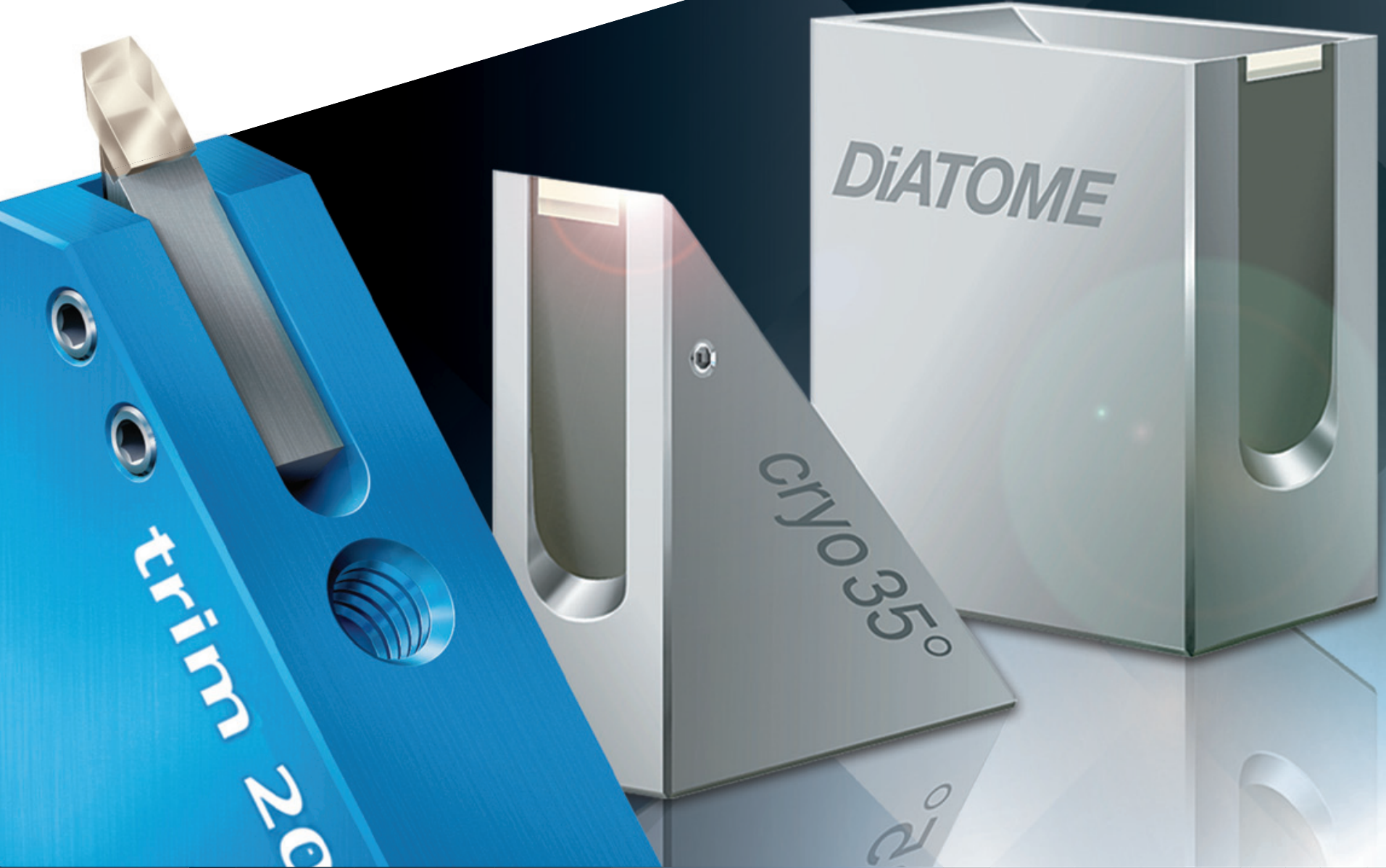

\section{DIATOME diamond knives}

ultra $45^{\circ} \cdot$ cryo $\cdot$ histo ultra $35^{\circ} \cdot$ histo jumbo cryo immuno • ultra sonic ultra AFM \& cryo AFM trimtool 20 - trimtool 45 trimtool 90

Over 40 years of development, manufacturing, and customer service

\section{DIATOME U.S.}

P.0. Box $550 \bullet 1560$ Industry Rd. • Hatfield, Pa 19440 Tel: (215) 412-8390 • Fax: (215) 412-8450

email: sgkcck@aol.com •www.emsdiasum.com

\section{Free customer service}

Sectioning tests with biological and material research specimens of all kinds. We send you the sections along with the surfaced sample, a report on the results obtained and a recommendation of a suitable knife. Complete discretion when working with proprietary samples.

Re-sharpening and reworking service

A re-sharpened Diatome diamond knife demonstrates the same high quality as a new knife. Even knives purchased in previous years can continue to be re-sharpened. The knives can be reworked into another type of knife for no extra charge, e.g. ultra to cryo or $45^{\circ}$ to $35^{\circ}$

\section{Exchange service}

Whenever you exchange a knife we offer you a new DiATOME knife at an advantageous price. 